\title{
Hypothalamic-pituitary-adrenal axis hypersensitivity in female rats on a post-weaning high-fat diet after chronic mild stress
}

\author{
LIAN LIU ${ }^{1,2}$, JUNQIANG YANG ${ }^{1,2}$, FENG QIAN ${ }^{2,3}$ and CHENGBIAO LU ${ }^{2,3}$ \\ ${ }^{1}$ Department of Pharmacology, Medical School of Yangtze University, Jingzhou, Hubei 434023; \\ ${ }^{2}$ Laboratory of Neuronal and Brain Disease Modulation, Yangtze University, Jingzhou, Hubei 434023; \\ ${ }^{3}$ Department of Physiology, Medical School of Yangtze University, Jingzhou, Hubei 434023, P.R. China
}

Received January 6, 2016; Accepted December 23, 2016

DOI: $10.3892 / \mathrm{etm} .2017 .4498$

\begin{abstract}
A high-fat diet (HFD) is highly correlated to obesity, metabolic diseases and certain behavioral changes. However, the effects of post-weaning HFD in rats during puberty and the role of the hypothalamic-pituitary-adrenal (HPA) axis in this process have remained elusive. The present study hypothesized that the HPA axis mediates the behavioral alterations induced by a post-weaning HFD. To investigate this, female rats were divided into two groups, one of which was fed a HFD from postnatal weeks (PWs) 4-12, while the other group received standard chow. Rats in each group were then subdivided into two subgroups each, and from PW 9-12, animals from one of the two subgroups were subjected to chronic mild stress (CMS), while the other subgroup received no stress. At PW 12, the body weight of rats receiving a HFD but no DMS was significantly higher than that in the control group. The frequency of crossing and rearing in the open field test and the time in the center of the Y-maze were decreased following CMS. Total time to escape was decreased in rats receiving HFD and after CMS. The serum levels of adrenocorticotropic hormone and corticosterone were increased in rats receiving an HFD and after CMS, and the mRNA levels of corticotropin-releasing hormone and arginine vasopressin in the hypothalamus were increased in the HFD + CMS group compared to that in the control group. The mRNA expression of glucocorticoid receptor (GR) in the hippocampi of rats in the HFD + CMS group was significantly decreased and the
\end{abstract}

Correspondence to: Dr Lian Liu, Department of Pharmacology, Medical School of Yangtze University, 1 Nan Huan Road, Jingzhou, Hubei 434023, P.R. China

E-mail: zifanqie_00@126.com

Dr Chengbiao Lu, Laboratory of Neuronal and Brain Disease Modulation, Yangtze University, 1 Nan Huan Road, Jingzhou, Hubei 434023, P.R. China

E-mail: johnlu9001@hotmail.com

Key words: hypersensitivity, hypothalamic-pituitary-adrenal axis, post-weaning, high-fat diet, behavioral alterations mineralocorticoid receptor/GR ratio was increased compared to that in the groups receiving either CMS or a HFD. In conclusion, these results indicated that female rats fed a post-weaning HFD showed HPA axis hypersensitivity under CMS, which may mediate behavioral alterations.

\section{Introduction}

A high-fat diet (HFD) is known to cause numerous metabolic diseases, such as obesity, insulin resistance and fatty liver. Exposure to a HFD at puberty increases the risk of type 2 diabetes, coronary heart disease and metabolic syndrome (1). Apart from atherosclerosis and obesity, HFD is also associated with neuroendocrine alterations (2). Obesity, which may develop as a result of a HFD, is known to increase the risk of behavioral disorders associated with anxiety in humans; this is particularly pronounced among females (3). A recent epidemiological survey revealed that behavioral and emotional problems during childhood are associated with pre- and postnatal HFD (4). Likewise, female adult rats exposed to a HFD for merely 1 week exhibited anxiety and reduced exploration behavior (5).

A HFD during adolescence has also been shown to increase the blood corticosterone (CORT) levels and expression of glucocorticoid receptors (GRs) in the hypothalamus and hippocampus $(6,7)$. The activity of the hypothalamic-pituitary-adrenal (HPA) axis is modulated by a feedback regulation of GCs and adrenocorticotropic hormone (ACTH) at the levels of the hippocampus, paraventricular nucleus of hypothalamus and pituitary gland. Verma et al (8) found an increased sensitivity of the adrenal cortex in women compared to men. In addition, the variance in ACTH and CORT levels in response to stress are uniformly higher in female rodents. The HPA axis is a vital neuroendocrine axis with a negative feedback regulation center in the hippocampus. The paraventricular nucleus of the hypothalamus directly modulates HPA axis activity (9). Multiple studies have demonstrated that HPA axis imbalance contributes to diseases of the central nervous system $(10,11)$. Clinical evidence has reveals that patients with depression or anxiety are also characterized by increased levels of corticotropin-releasing hormone (CRH) and arginine vasopressin (AVP) (12). A HFD is also associated with HPA axis alterations. For instance, maternal HFD during pregnancy 
modulates glucocorticoid signaling pathways in limbic areas, and is associated with the regulation of HPA function and anxiety in adulthood (13). A post-weaning HFD until early puberty leads to altered HPA axis function in female rats, indicating that HFD-induced nutritional imbalance in young females may lead to neuroendocrine dysfunction, which in turn may promote the appearance of stress-associated disorders during adolescence (6). Chronic mild stress (CMS) is often associated to the pathogenesis of obesity (14). The ability of CMS to lead to depression, including the wide range of accompanying physical, neurochemical and behavioral changes (e.g., anhedonia) have been well documented (15-17).

Few experimental animal studies on CMS have induced an obese-like state in the animals. Instead, CMS leads to decreases in the consumption of highly palatable foods with subsequent loss in body weight (18-20). Due to the inherent variability of stress paradigms, it is difficult to map out the hormonal and neurochemical responses to CMS. In any given CMS protocol, multiple stressors are used. Furthermore, it is difficult to draw parallels between animal models of CMS and any type of chronic stress in humans (21).

Thus far, however, the effects of a HFD from post-weaning age until adolescence on the HPA axis and behavior have remained elusive. Moreover, the association between HFD-induced behavioral changes and HPA axis sensitivity has remained to be determined. Therefore, the present study used a female rat model to assess the effects of a post-weaning HFD, CMS and their combination on the HPA axis as well as their behavior. Furthermore, the hippocampal mineralocorticoid receptor (MR) and glucocorticoid receptor (GR) expression levels were determined in order to assess any possible correlation between the HFD-induced altered HPA axis sensitivity and behavioral changes.

\section{Materials and methods}

Animals and treatment. Animal experiments were performed at the Animal Center of Yangtze University (Jingzhou, China), which is accredited by the Association for Assessment and Accreditation of Laboratory Animal Care International. The study protocol was designed in accordance with the Guidelines for the Care and Use of Laboratory Animals and was approved by the Ethical Research Committee of the Medical College of Yangtze University (Jingzhou, China). Wistar rats (8-10 weeks; $\mathrm{n}=8$ male and 8 female) were supplied the by Experimental Center of Hubei Medical Scientific Academy (no. 2009-0004; Hubei, China). The animals were housed under standard conditions (temperature, $18-22^{\circ} \mathrm{C}$; humidity, 40-60\%; 12:12 h light-dark cycle) and allowed access to food ad libitum.

The animals were treated as described previously (22). Pregnant rats were fed independently until delivery, and a total of 8 pups from each litter were kept ( 4 males and 4 females). On postnatal week (PW) 4, the pups were separated from their mothers, and 16 female pups ( 2 female pups from each mother) were assigned to each group. One group was fed a normal diet (21\% kcal from protein, $68.5 \% \mathrm{kcal}$ from carbohydrates and $10.5 \%$ kcal from fat) and the other group received a HFD (88.0\% corn flour, $11.5 \%$ lard and $0.5 \%$ cholesterol, which provided $18.9 \% \mathrm{kcal}$ from protein, $61.7 \% \mathrm{kcal}$ from carbohydrates and $19.4 \%$ kcal from fat) (23). The HFD and control groups were then further subdivided into two subgroups each ( $n=8$ in each): One subgroup of each group was subjected to 21 days of CMS from PWs 9-12, while the second subgroup was not subjected to stress. Thus, there were a total of four subgroups ( $n=8$ rats per group): The control, in which the rats were fed standard chow and received no stress; the HFD group, in which the rats were fed an HFD; the CMS group, in which the rats were subjected to CMS; and the CMS + HFD group, in which the rats received a HFD as well as CMS. Body weights were determined every 2 weeks from PW4-12.

At PW8, all the animals were subjected to the open field test and electric maze test (Fig. 1). The time interval between the sequential tests was two days. Behavioral tests were performed between 09:30 am and 15:30 pm. Blood samples were collected from the caudal vein after the rats were made to fast for $12 \mathrm{~h}$. The rats in the CMS and CMS + HFD groups were subjected to CMS from PW9-12 according to the procedure described in a previous study (23). This included food and water deprivation for $24 \mathrm{~h}$, pinching of the tail $(2 \mathrm{~cm}$ from the tail end) for $5 \mathrm{~min}$, treatment with heat at $45^{\circ} \mathrm{C}$ for $5 \mathrm{~min}$, swimming in cold water at $4-8^{\circ} \mathrm{C}$ for 4 min followed by towel drying, reversal of the day and night cycles and social isolation (single rat per cage) for $24 \mathrm{~h}$. The stressors were randomly administered and continuously applied at day and night. On PW13, after the last open field test and electric maze test, all of the animals were anesthetized with isoflurane (Baxter Healthcare Co., Deerfield, IL, USA) and decapitated prior to blood sample collection. Finally, the hippocampi and hypothalami were dissected and stored at $-80^{\circ} \mathrm{C}$ for subsequent use.

\section{Behavioral tests}

Open field test. According to the protocol of a previous study (24), the open field test was used to evaluate the locomotor activity and exploratory behavior of the rats. A plywood box with black walls and the dimensions 120x90x30 cm was divided into 25 equal squares. Each rat was individually placed in the center of the apparatus at the time of the test for $3 \mathrm{~min}$ and their behavior during this time were recorded in detail. When the hind legs crossed the line of a square, the rat was considered to have crossed over to the next square (crossing), and when the forelegs were lifted from the floor, the animal scored one point. At the end of each open field test, the cage was carefully cleaned with $75 \%$ ethanol and purified water prior to the test for the next rat, ensuring no possible interference with the spontaneous behavior of the next animal.

Electric maze test. According to a previously described method (22), rat learning and memory were determined by using a Y-type electric maze test. In brief, each rat was placed in the maze and electrical stimulation was performed. Upon electrical stimulation, direct escape from the starting position to a safe region was considered as the correct reaction. The times of correct reaction (TOCR) and total time to escape (TTE) in 10 tests were utilized to evaluate learning and memory abilities.

Determination of serum ACTH and CORT levels. The serum ACTH and CORT levels were determined according to previously described methods (25) using radioimmunoassay (RIA) and ELISA kits in accordance with the manufacturers' 
Table I. Primers and annealing conditions.

\begin{tabular}{lllc} 
Gene & \multicolumn{1}{c}{ Forward primer $\left(5^{\prime}-3^{\prime}\right)$} & \multicolumn{1}{c}{ Reverse primer $\left(5^{\prime}-3^{\prime}\right)$} & $\begin{array}{c}\text { Annealing temperature } \\
\left({ }^{\circ} \mathrm{C}\right) / \text { time }(\mathrm{sec})\end{array}$ \\
\hline$\beta$-actin & GTTGCCAATAGTGATGACCT & GGACCTGACAGACTACCTCA & $54 / 20$ \\
CRH & AGAACAACAGTGCGGGCTCA & GCTCCGGTTGCAAGAAATTCA & $60 / 30$ \\
AVP & AAGAGGGCCACATCCGACA & AGGGCAGGTAGTTCTCCTCCTG & $58 / 20$ \\
MR & TGCATGATCTCGTGAGTGA & AAGTTCTTCCTGGCCGGTAT & $62 / 30$ \\
GR & CACCCATGACCCTGTCAGTC & AAAGCCTCCCTCTGCTAACC & $61 / 30$
\end{tabular}

CRH, corticotropin-releasing hormone; AVP, arginine vasopressin; MR, mineralocorticoid receptor; GR, glucocorticoid receptor.



Figure 1. Schematic illustration of the course of animal treatment in the present study. PW, postnatal weeks.

protocols. The rat CORT ELISA kit was obtained from Assaypro (St. Charles, MO, USA). The rat ACTH RIA kit was obtained from Beijing North Biotech Institute (Beijing, China). The gain rate of serum ACTH and CORT levels after vs. prior to stress were calculated as follows: Gain rate $(\%)=[$ (Concentration after stress-concentration before stress)/concentration before stress] x 100 .

Reverse-transcription quantitative polymerase chain reaction $(R T-q P C R)$. RT-qPCR was performed to assess the mRNA expression levels of MR and GR in the hippocampus as well as CRH and AVP in the hypothalamus. Oligonucleotide primers used in the present study for PCR were all synthesized by Sangon Biotech Co., Ltd. (Shanghai, China) and are listed in Table I. Total RNA was isolated using TRIzol reagent (Invitrogen; Thermo Fisher Scientific, Inc., Waltham, MA, USA) according to the manufacturer's instructions, and its concentration and purity were determined with a spectrophotometer. Total RNA was stored in diethyl pyrocarbonate-treated $\mathrm{H}_{2} \mathrm{O}\left(\right.$ DEPC- $\left.\mathrm{H}_{2} \mathrm{O}\right)$ at $-80^{\circ} \mathrm{C}$. RT-qPCR analysis was performed using a reverse transcription and qPCR kit (Takara Biotechnology Co., Ltd., Dalian, China), and reactions were performed and products were analyzed using an ABI PRISM 7300 instrument (Applied Biosystems; Thermo Fisher Scientific, Inc.). Single-stranded complementary (c)DNA was prepared according to the protocol of the Exscript RT reagent kit (Takara Biotechnology Co., Ltd.). The amplification system had a total reaction volume of $25 \mu$ l containing
$2 \mu \mathrm{l}$ of a $0.1 \mu \mathrm{g} / \mu 1$ solution of cDNA template, $0.5 \mu \mathrm{l}$ of a $10 \mu \mathrm{mol} / 1$ solution of each primer, $12.5 \mu 12 \mathrm{X}$ Premix Ex Taq, $0.5 \mu 1$ 20X SYBR-Green I and $9 \mu 1$ DEPC- $\mathrm{H}_{2} \mathrm{O}$. The mRNA levels of the housekeeping gene $\beta$-actin were measured as quantitative controls and each sample was normalized to the specific $\beta$-actin mRNA content (26). PCR amplification was performed using the following protocol: Initial denaturation step at $95^{\circ} \mathrm{C}$ for $5 \mathrm{~min} ; 40$ cycles of $95^{\circ} \mathrm{C}$ for $5 \mathrm{sec}$, with various annealing conditions (Table I) and a step at $72^{\circ} \mathrm{C}$ for $30 \mathrm{sec}$ if the annealing temperature was $<60^{\circ} \mathrm{C}$.

Statistical analysis. Data were analyzed using SPSS 17 (SPSS Inc., Chicago, IL, USA) and GraphPad Prism 6 (GraphPad Software, Inc., La Jolla, CA, USA). Values are expressed as the mean \pm standard error of the mean. Analysis with Student's unpaired t-test was used to compare the mean of different groups, as applicable. Analysis with a paired t-test was used to compare the means of the same group before and after chronic stress, assuming equal variances. $\mathrm{P}<0.05$ was considered to indicate a statistically significant difference.

\section{Results}

HFD from PW 4-12 increases rat body weight gain. As shown in Table II, there were no significant differences in the body weight between the HFD, CMS, HFD + CMS and control groups before PW 10. The body weight in the HFD group was significantly increased at PW 12 compared with that in the 
control group $(\mathrm{P}<0.01)$. The body weight in the HFD + CMS group at PW 12 as well as that in the HFD group at PWs 6,8 and 10 was also increased relative to the control at the respective time-points; however, the differences were not statistically significant.

Post-weaning HFD and CMS induce behavioral alterations in female rats. In the open field test, CMS significantly decreased the frequency of crossing in rats receiving a normal diet or HFD ( $\mathrm{P}<0.01$; Fig. 2A and B). Furthermore, after CMS, the frequency of crossing and rearing in the rats receiving a HFD was lower compared to that in rats receiving a normal diet $(\mathrm{P}<0.05)$. However, there were no significant changes in the frequency of grooming, total distance moved and distance moved from the center between the HFD and HFD + CMS groups (Fig. 2C-E). Of note, CMS significantly reduced the time spent in the central zone of the maze by rats in rats receiving an $\mathrm{HFD}(\mathrm{P}<0.05$; Fig. $2 \mathrm{~F})$.

The rats were subjected to the electric maze test to assess animal behavior after stress. Prior to CMS, there were no differences in reaction time and the TTE between the HFD and control groups. However, after CMS, the TOCR was significantly decreased $(\mathrm{P}<0.05)$ and the total TTE was slightly increased in the HFD group relative that in the control group (Table III). The TOCR of both the control and HFD groups were significantly decreased after CMS $(\mathrm{P}<0.01)$. However, the change in the HFD group after CMS was greater than that in the control. The total TTE did not markedly change.

Post-weaning HFD and CMS affect serum ACTH and CORT levels in female rats. Prior to CMS, the serum ACTH levels were similar between the control and HFD groups, while the CORT levels were increased in the HFD group $(\mathrm{P}<0.05)$. After CMS, the group receiving the HFD showed higher serum $\mathrm{ACTH}$ and CORT levels than the group on a normal diet $(\mathrm{P}<0.01$; Fig. $3 \mathrm{~A}$ and $\mathrm{B})$. Furthermore, in the rats receiving a normal diet as well as a HFD, the ACTH $(\mathrm{P}<0.01)$ and CORT $(\mathrm{P}<0.05$ and $\mathrm{P}<0.01)$ levels were elevated after CMS, while the CMS-induced increases in the levels of these hormones were considerably higher in the HFD rats compared with those in the rats receiving a normal diet $(\mathrm{P}<0.05$; Fig. 3C).

Post-weaning HFD and CMS affect hypothalamic CRH and AVP mRNA levels in female rats. The AVP and CRH mRNA levels in the hypothalamus were significantly increased in the HFD + CMS group $(\mathrm{P}<0.05$ and $\mathrm{P}<0.01$, respectively $)$ compared to those in the control group (Fig. 4A and B). In addition, the CRH mRNA levels in the hypothalamus were significantly increased in the HFD group compared with those in the control group $(\mathrm{P}<0.01)$. Furthermore, CMS significantly increased the CRH mRNA levels in the hypothalamus compared with those in the control group ( $\mathrm{P}<0.05$; Fig. 4B). Finally, the CRH mRNA levels in the hypothalamus were higher in the HFD+CMS group than those in the CMS group $(\mathrm{P}<0.05)$.

Effects of post-weaning HFD and CMS on hippocampal MR and GR mRNA expression levels in female rats. No significant 
Table III. TOCR and TTE of the female rats in the electric maze test prior to and after CMS.

\begin{tabular}{lccccc}
\hline & \multicolumn{2}{c}{ Before CMS } & & \multicolumn{2}{c}{ After CMS } \\
\cline { 2 - 3 } Group & TOCR (count) & & TOCR (count) & TTE (sec) \\
\hline Control & $7.62 \pm 0.37$ & $65.98 \pm 4.96$ & & $6.00 \pm 0.76^{\mathrm{a}}$ & $78.44 \pm 4.91$ \\
HFD & $7.12 \pm 0.29$ & $64.92 \pm 9.34$ & & $4.75 \pm 1.03^{\mathrm{a}, \mathrm{b}}$ & $87.21 \pm 5.12$
\end{tabular}

${ }^{a} \mathrm{P}<0.01$ vs. before CMS by paired t-test; ${ }^{b} \mathrm{P}<0.05$ vs. control by independent $\mathrm{t}$-test. Values are expressed as the mean \pm standard error of the mean $(n=8)$. Rats in the HFD group were fed a HFD containing $24 \%$ fat by weight from PW 4-12. A proportion of the rats were subjected to 21 days of CMS from PW 9-12. TOCR, times of correct reaction; TTE, total time to escape; HFD, high-fat diet; CMS, chronic mild stress; PW, postnatal week.

A
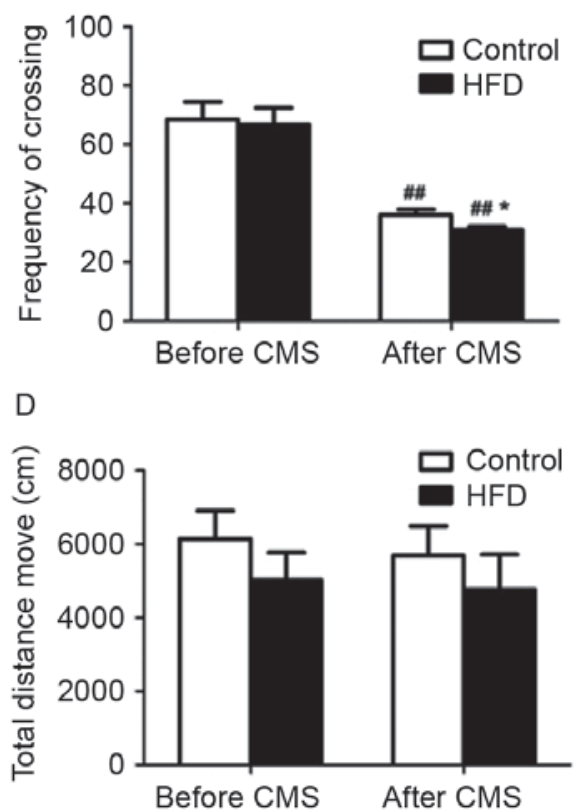

B
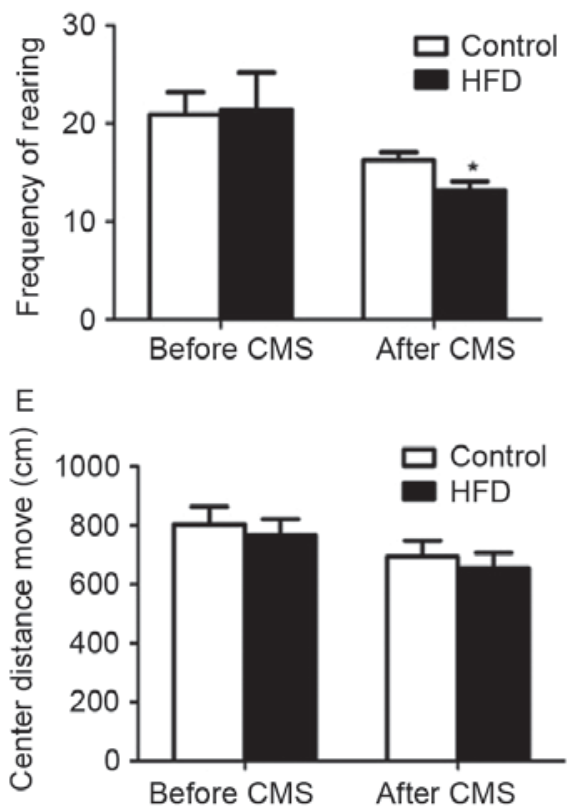

C



$\mathrm{F}$

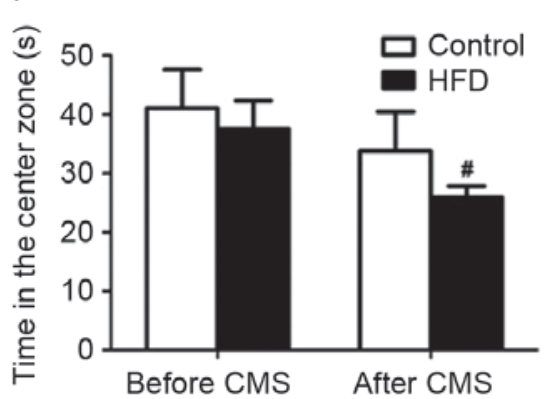

Figure 2. Effects of a post-weaning HFD on frequencies of (A) crossing, (B) rearing and (C) grooming, as well as (D) total distance, (E) center distance and (F) time spent in the central area by female rats in the open field test prior to and after induction of CMS. The female rats from the HFD group were fed a HFD containing $24 \%$ fat by weight from PWs 4-12. A proportion of the animals were subjected to 21 days of CMS at PW 9-12. Values are expressed as the mean \pm standard error of the mean $(n=8)$. ${ }^{*} \mathrm{P}<0.05$ vs. the control group (Student's unpaired t-test); ${ }^{~} \mathrm{P}<0.05$ and ${ }^{\# \#} \mathrm{P}<0.01$ vs. before CMS (paired t-test). HFD, high-fat diet; CMS, chronic mild stress; PW, postnatal weeks.

changes were observed in the hippocampal MR and GR mRNA levels between the HFD and control groups (Fig. 5A and $\mathrm{B}$ ). Among all of the groups, no significant changes in the hippocampal MR mRNA expression were detected. However, the GR gene expression levels in the hippocampus were significantly lower in the HFD + CMS group than in the HFD and CMS groups (all $\mathrm{P}<0.05$; Fig. 5B); in addition, the MR/GR ratio in the HFD + CMS group was overtly increased $(\mathrm{P}<0.05$; Fig. 5C).

\section{Discussion}

The high-fat rat diet used in the present study ( $24 \%$ fat) mimics the HFD consumed by humans $(6,7)$. Previous studies have shown that women are more prone to mental diseases after HFD and exhibit a higher sensitivity to CMS than men (27), which is why only female rats were assessed in the present study. Fat accumulation is more pronounced when energy is gained from dietary fat rather than from carbohydrates or proteins (28). In the present study, the rat body weight did not show any significant difference between the HFD and control groups at PW 10, while the increase was significant at PW 12. While HFD obviously induced the increase in body weight, it may have also in part been attributed to enhanced food intake, metabolic disturbance of adipocytes and energy imbalance, eventually leading to an obesity-like state (29). Of note, there was no significant body weight change between the CMS and control groups, indicating that CMS alone did not induce any changes in weight gain, indicating that CMS alone without a HFD does not lead to obesity.

Behavioral alterations are a major symptom of mental disorders $(30,31)$. These behavioral alterations are induced by regulatory dysfunction of the cerebral limbic system (32). A maternal HFD has been shown to lead to anxiety in offspring, 

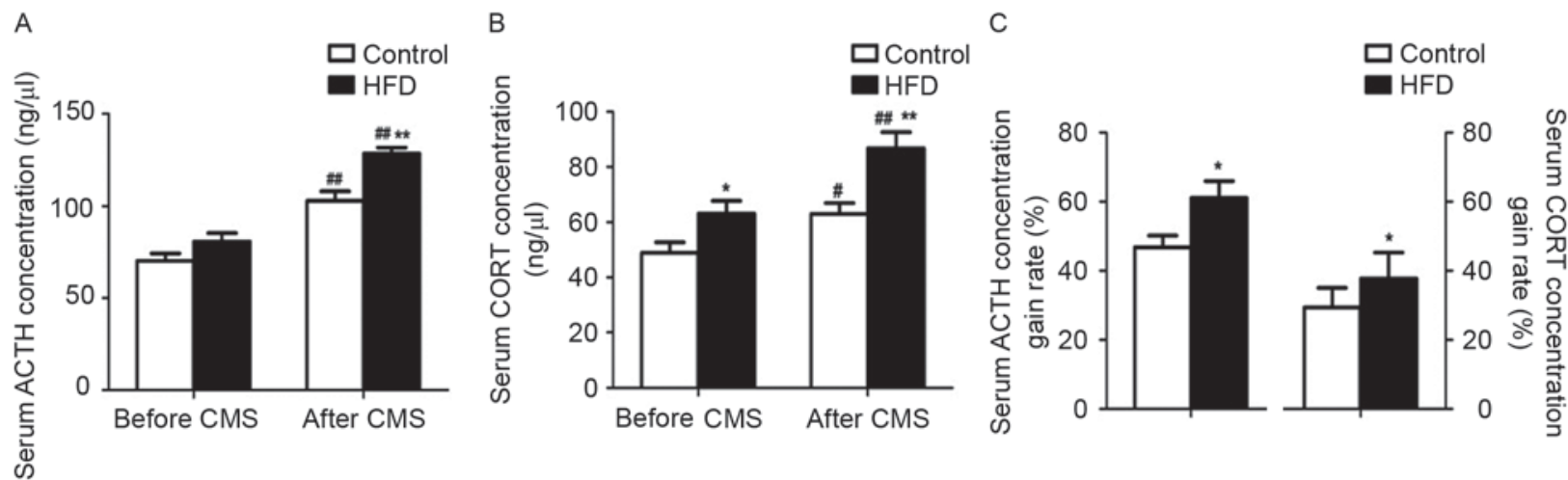

Figure 3. Effects of a post-weaning HFD on the serum (A) ACTH and (B) CORT levels prior to and after CMS were detected by radioimmunoassay and ELISA, respectively. Rats in the HFD group were fed a HFD containing $24 \%$ fat by weight from PW 4-12. A proportion of the rats were subjected to 21 days of CMS from PW 9-12. (C) The gain rates of ACTH and CORT were also calculated. Values are expressed as the mean \pm standard error of the mean $(\mathrm{n}=8)$. ${ }^{\mathrm{P}}<0.05$, ${ }^{* *} \mathrm{P}<0.01$ vs. control group (Student's unpaired t-test); ${ }^{\#} \mathrm{P}<0.05,{ }^{\# \#} \mathrm{P}<0.01$ vs. before CMS (paired t-test). ACTH, adrenocorticotropin-releasing hormone; CORT, corticosterone; HFD, high-fat diet; CMS, chronic mild stress; PW, postnatal weeks.
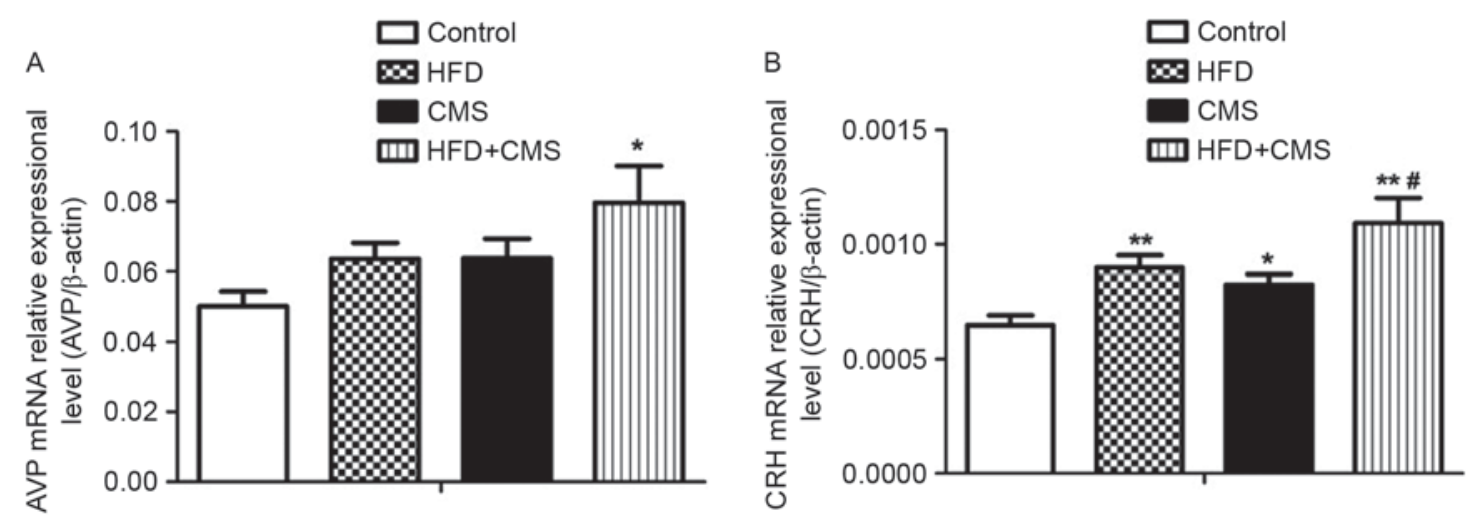

Figure 4. Effects of a post-weaning HFD on gene expression levels of hypothalamic (A) CRH and (B) AVP in the control, HFD, CMS and CMS + HFD groups at PW 13. Values are expressed as the mean \pm standard error of the mean $(\mathrm{n}=8)$. ${ }^{*} \mathrm{P}<0.05$ and ${ }^{* * *} \mathrm{P}<0.01$ vs. control; ${ }^{*} \mathrm{P}<0.05$ vs. CMS. Groups: Control, rats receiving normal diet and not subjected to CMS; CMS, rats receiving normal diet and subjected to CMS (at PW 9-12); HFD, rats receiving HFD (24\% fat by weight from PW 4-12) but not subjected to CMS; CMS + HFD, rats receiving a HFD and subjected to CMS. CRH, corticotropin-releasing hormone; AVP, arginine vasopressin; HFD, high-fat diet; CMS, chronic mild stress; PW, postnatal weeks.
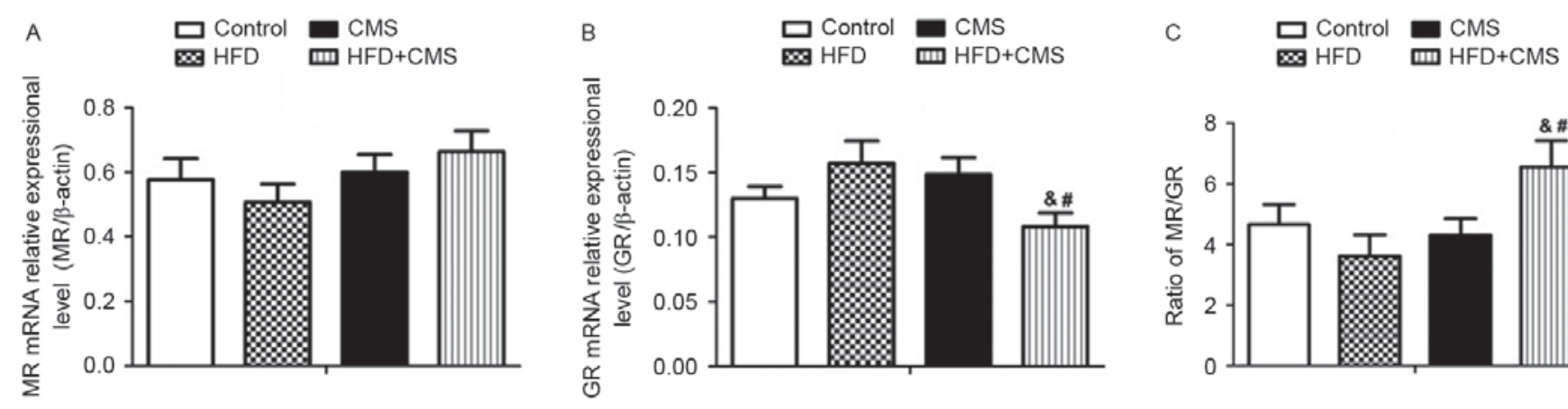

Figure 5. Effects of post-weaning HFD on hippocampal expression levels of (A) MR, (B) GR and (C) the ration of MR/GR in the control, HFD, CMS and $\mathrm{CMS}+\mathrm{HFD}$ groups at PW 13. Values are expressed as the mean \pm standard error of the mean $(\mathrm{n}=8) .{ }^{\text {* }} \mathrm{P}<0.05$ vs. HFD group; ${ }^{*} \mathrm{P}<0.05$ vs. CMS group. Groups: Control, rats receiving normal diet and not subjected to CMS; CMS, rats receiving normal diet and subjected to CMS (at PW 9-12); HFD, rats receiving HFD (24\% fat by weight from PW 4-12) but not subjected to CMS; CMS + HFD, rats receiving a HFD and subjected to CMS. HFD, high-fat diet; CMS, chronic mild stress; PW, postnatal weeks; MR, mineralocorticoid receptor; GR, glucocorticoid receptor.

altering the expression levels of multiple genes via glucocorticoid signaling (33). HFD was shown to have rapid and long-lasting negative effects on memory in model mice with and without Alzheimer's disease (34). The open field test is a classic experimental method for behavioral evaluation, including locomotor activity and exploratory behavior, and the electric maze test is used to assess learning and memory. In the open field test performed in the present study, application of CMS 
resulted in a significantly reduced frequency of crossings in rats with and without HFD as well as a decreasing tendency in the frequency of rearing, and the time in the center zone in the HFD group significantly reduced following CMS, suggesting depression-like symptoms after CMS. The electric maze test revealed that CMS significantly reduced the TOCR of mice with or without HFD and increased the TTE, particularly in the HFD group, suggesting that post-weaning HFD not only predisposed female rats to psychiatric disorders but also altered learning and memory in them. Further study is required to confirm whether HFD may alter learning and memory. Taken together, HFD and CMS appear to act synergistically to cause depression-like symptoms. Indeed, multiple studies have suggested that CMS causes depression-like behaviors, such as alterations of affective processes and impairment of cognitive performance (35).

The HPA axis has a vital role in the stress response and is affected by a number of factors (36-38). Animal experiments have shown that food restriction increased adrenal gland weight and serum CORT levels after overnight fasting in rats (36), and enhanced HPA axis activity resulting in appetite loss and anorexia nervosa (39). Mixed results have been reported by studies assessing the effects of HFD exposure on CORT during developmental stages under basal conditions and after stress challenge $(2,40)$. In the present study, CMS resulted in higher ACTH and CORT levels and higher expression of CRH gene in hypothalamus tissue in the HFD group compared to that in the control group, suggesting that HFD may enhance HPA axis sensitivity to chronic stress. Considerable evidence suggested associations between certain psychiatric disorders and HPA axis alterations, such as elevated glucocorticoid levels (10,41-43). Furthermore, several psychiatric disorders associated with HPA dysfunction arise in obese teenagers, who are more prone to develop depression than adults (44). Therefore, it can be speculated that behavioral alterations in female rats with post-weaning HFD may be attributed to hypersensitivity of the HPA axis induced by HFD.

The hippocampus is the main negative feedback regulation center of the HPA axis. MR and GR are the steroid receptors predominantly expressed in the hippocampus. The hippocampus regulates HPA function in a negative feedback pattern via the binding of circulating GCs to hippocampal GR and MR. These two receptors have different roles in HPA axis regulation under different conditions. Under basal conditions, the hippocampal MR is mainly responsible for the regulation of the HPA axis by glucocorticoids, while GR becomes the major receptor for glucocorticoid under stress (45). Activation of these receptors can modulate the neuronal system associated with function including memory, behavior, responses to stimuli and anxiety (45). MR harbors a neuroprotective function in the hippocampus (46), while GR is a typical target of neuronal signaling that is sensitive and vulnerable to GC and its over-activation would damage hippocampal neurons $(45,47)$, which may lead to behavioral alterations. For instance, HFD administered from post-weaning age until puberty was found to decrease the hippocampal GR protein expression in female rats (6). In the present study, hippocampal MR mRNA levels showed an increasing tendency after CMS, while GR expression was significantly reduced in the HFD group, increasing the $\mathrm{MR} / \mathrm{GR}$ ratio. Zhe et al (48) demonstrated that stress induced downregulation of MR and GR and the MR/GR ratio in the hippocampus. MR carries neuron-protective function in the hippocampus; the increase in MR mRNA in hippocampus is involved in negative feedback. However, to the best of our knowledge, there have been no reports on the association between the HFD and the hippocampal MR expression. A change in MR/GR ratio alters the ability to maintain homeostasis, which, in turn, alters neuronal excitability, stress responsiveness and behavioral adaptation to a condition of enhanced vulnerability to disease (49). Therefore, it can be speculated that dysregulation of the MR/GR balance may aggravate HPA axis hypersensitivity, which may be an important cause of behavioral alterations.

In conclusion, the present study revealed that post-weaning HFD increased HPA activity in female rats, and the combination of HFD and CMS enhanced those effects. In addition, female rats that were fed a HFD exhibited impaired memory and learning skills following CMS. These effects may be due to hippocampal MR/GR dysregulation, which further aggravated HPA axis hypersensitivity.

\section{Acknowledgements}

This work was supported by grants from the Science Foundation of Hubei Provincial Department of Education (grant nos. Q20151308 and Q20141301), the Yangtze Fund for Youth Teams of Science and Technology Innovation (grant no. 2016YZYT1128), Hubei Province Health and Family Planning Scientific Research Project (grant no. WJ2016-YZ-01), the Medical School of Yangtze University Foundation (grant nos. YXYQ201401 and YXYQ201405).

\section{References}

1. Peleg-Raibstein D, Luca E and Wolfrum C: Maternal high-fat diet in mice programs emotional behavior in adulthood. Behav Brain Res 233: 398-404, 2012.

2. Auvinen HE, Romijn JA, Biermasz NR, Pijl H, Havekes LM, Smit JW, Rensen PC and Pereira AM: The effects of high fat diet on the basal activity of the hypothalamus-pituitary-adrenal axis in mice. J Endocrinol 214: 191-197, 2012.

3. Rofey DL, Kolko RP, Iosif AM, Silk JS, Bost JE, Feng W, Szigethy EM, Noll RB, Ryan ND and Dahl RE: A longitudinal study of childhood depression and anxiety in relation to weight gain. Child Psychiatry Hum Dev 40: 517-526, 2009.

4. O'Connor TG: Prenatal and postnatal exposure to an unhealthy diet is associated with behavioural and emotional problems in children. Evid Based Ment Health 17: 38, 2014.

5. Soulis G, Papalexi E, Kittas C and Kitraki E: Early impact of a fat-enriched diet on behavioral responses of male and female rats. Behav Neurosci 121: 483-490, 2007.

6. Boukouvalas G, Antoniou K, Papalexi E and Kitraki E: Post weaning high fat feeding affects rats' behavior and hypothalamic pituitary adrenal axis at the onset of puberty in a sexually dimorphic manner. Neuroscience 153: 373-382, 2008.

7. Boukouvalas G, Gerozissis $\mathrm{K}$ and Kitraki E: Adult consequences of post-weaning high fat feeding on the limbic-HPA axis of female rats. Cell Mol Neurobiol 30: 521-530, 2010.

8. Verma R, Balhara YP and Gupta CS: Gender differences in stress response: Role of developmental and biological determinants. Ind Psychiatry J 20: 4-10, 2011.

9. Szabo K and Hennerici MG (eds): The Hippocampus in Clinical Neuroscience. Front Neurol Neurosci Basel Karger, 2014.

10. Li SX, Yan SY, Bao YP, Lian Z, Qu Z, Wu YP and Liu ZM: Depression and alterations in hypothalamic-pituitary-adrenal and hypothalamic-pituitary-thyroid axis function in male abstinent methamphetamine abusers. Hum Psychopharmacol 28: 477-483, 2013 
11. Moreno-Ramos OA, Lattig MC and González Barrios AF: Modeling of the hypothalamic-pituitary-adrenal axis-mediated interaction between the serotonin regulation pathway and the stress response using a Boolean approximation: A novel study of depression. Theor Biol Med Model 10: 59, 2013.

12. Holsboer F and Ising M: Central CRH system in depression and anxiety-evidence from clinical studies with CRH1 receptor antagonists. Eur J Pharmacol 583: 350-357, 2008.

13. Sasaki A, de Vega WC, St-Cyr S, Pan P and McGowan PO Perinatal high fat diet alters glucocorticoid signaling and anxiety behavior in adulthood. Neuroscience 240: 1-12, 2013.

14. García-Díaz DF, Campion J, Milagro FI, Lomba A, Marzo F and Martínez JA: Chronic mild stress induces variations in locomotive behavior and metabolic rates in high fat fed rats. J Physiol Biochem 63: 337-346, 2007.

15. Stöhr T, Szuran T, Welzl H, Pliska V, Feldon J and Pryce CR: Lewis/Fischer rat strain differences in endocrine and behavioural responses to environmental challenge. Pharmacol Biochem Behav 67: 809-819, 2000.

16. Willner P: Validity, reliability and utility of the chronic mild stress model of depression: A 10-year review and evaluation. Psychopharmacology (Berl) 134: 319-329, 1997.

17. Willner P, Muscat R and Papp M: Chronic mild stress-induced anhedonia: A realistic animal model of depression. Neurosci Biobehav Rev 16: 525-534, 1992.

18. Muscat R and Willner P: Suppression of sucrose drinking by chronic mild unpredictable stress: A methodological analysis. Neurosci Biobehav Rev 16: 507-517, 1992.

19. Patterson ZR, Ducharme R, Anisman H and Abizaid A: Altered metabolic and neurochemical responses to chronic unpredictable stressors in ghrelin receptor-deficient mice. Eur J Neurosci 32: 632-639, 2010.

20. Westenbroek C, Ter Horst GJ, Roos MH, Kuipers SD, Trentani A and den Boer JA: Gender-specific effects of social housing in rats after chronic mild stress exposure. Prog Neuropsychopharmacol Biol Psychiatry 27: 21-30, 2003.

21. Patterson ZR and Abizaid A: Stress induced obesity: Lessons from rodent models of stress. Front Neurosci 7: 130, 2013.

22. Hou DR, Wang Y, Zhou L, Chen K, Tian Y, Song Z, Bao J and Yang QD: Altered angiotensin-converting enzyme and its effects on the brain in a rat model of Alzheimer disease. Chin Med J (Engl) 121: 2320-2323, 2008

23. Zhang L, Xu D, Zhang B, Liu Y, Chu F, Guo Y, Gong J, Zheng X, Chen L and Wang H: Prenatal food restriction induces a hypothalamic-pituitary-adrenocortical axis-associated neuroendocrine metabolic programmed alteration in adult offspring rats. Arch Med Res 44: 335-345, 2013

24. Xinxing W, Wei L, Lei W, Rui Z, Baoying J and Lingjia Q: A neuroendocrine mechanism of co-morbidity of depression-like behavior and myocardial injury in rats. PLoS One 9: e88427, 2014.

25. Liu L, Liu F, Kou H, Zhang BJ, Xu D, Chen B, Chen LB Magdalou J and Wang H: Prenatal nicotine exposure induced a hypothalamic-pituitary-adrenal axis-associated neuroendocrine metabolic programmed alteration in intrauterine growth retardation offspring rats. Toxicol Lett 214: 307-313, 2012.

26. Livak KJ and Schmittgen TD: Analysis of relative gene expression data using real-time quantitative PCR and the 2(-Delta Delta C(T)) Method. Methods 25: 402-408, 2001

27. Bao AM, Meynen G and Swaab DF: The stress system in depression and neurodegeneration: Focus on the human hypothalamus. Brain Res Rev 57: 531-553, 2008.

28. Woods SC, Seeley RJ, Rushing PA, D'Alessio D and Tso P: A controlled high-fat diet induces an obese syndrome in rats. J Nutr 133: 1081-1087, 2003.

29. McAllan L, Keane D, Schellekens H, Roche HM, Korpela R, Cryan JF and Nilaweera KN: Whey protein isolate counteracts the effects of a high-fat diet on energy intake and hypothalamic and adipose tissue expression of energy balance-related genes. $\mathrm{Br}$ J Nutr 110: 2114-2126, 2013.

30. Nelovkov A, Philips MA, Kõks S and Vasar E: Rats with low exploratory activity in the elevated plus-maze have the increased expression of limbic system-associated membrane protein gene in the periaqueductal grey. Neurosci Lett 352: 179-182, 2003.
31. Hoogenboom WS, Perlis RH, Smoller JW, Zeng-Treitler Q, Gainer VS, Murphy SN, Churchill SE, Kohane IS, Shenton ME and Iosifescu DV: Limbic system white matter microstructure and long-term treatment outcome in major depressive disorder: A diffusion tensor imaging study using legacy data. World J Biol Psychiatry 15: 122-134, 2014.

32. Winter SS, Köppen JR, Ebert TB and Wallace DG: Limbic system structures differentially contribute to exploratory trip organization of the rat. Hippocampus 23: 139-152, 2013.

33. Sasaki A, de Vega W, Sivanathan S, St-Cyr S and McGowan PO: Maternal high-fat diet alters anxiety behavior and glucocorticoid signaling in adolescent offspring. Neuroscience 272: 92-101, 2014.

34. Knight EM, Martins IV, Gümüsgöz S, Allan SM and Lawrence CB: High-fat diet-induced memory impairment in triple-transgenic Alzheimer's disease (3xTgAD) mice is independent of changes in amyloid and tau pathology. Neurobiol Aging 35: 1821-1832, 2014.

35. Sahin TD, Karson A, Balci F, Yazir Y, Bayramgurler D and Utkan T: TNF-alpha inhibition prevents cognitive decline and maintains hippocampal BDNF levels in the unpredictable chronic mild stress rat model of depression. Behav Brain Res 292: 233-240, 2015.

36. Belda X, Ons S, Carrasco J and Armario A: The effects of chronic food restriction on hypothalamic-pituitary-adrenal activity depend on morning versus evening availability of food. Pharmacol Biochem Behav 81: 41-46, 2005

37. Kokavec A, Lindner AJ, Ryan JE and Crowe SF: Ingesting alcohol prior to food can alter the activity of the hypothalamic-pituitary-adrenal axis. Pharmacol Biochem Behav 93: 170-176, 2009.

38. Naert G, Ixart G, Maurice T, Tapia-Arancibia L and Givalois L: Brain-derived neurotrophic factor and hypothalamic-pituitary-adrenal axis adaptation processes in a depressive-like state induced by chronic restraint stress. Mol Cell Neurosci 46: 55-66, 2011.

39. Lawson EA, Holsen LM, Desanti R, Santin M, Meenaghan E, Herzog DB, Goldstein JM and Klibanski A: Increased hypothalamic-pituitary-adrenal drive is associated with decreased appetite and hypoactivation of food-motivation neurocircuitry in anorexia nervosa. Eur J Endocrinol 169: 639-647, 2013.

40. Shalev U, Tylor A, Schuster K, Frate C, Tobin S and Woodside B: Long-term physiological and behavioral effects of exposure to a highly palatable diet during the perinatal and post-weaning periods. Physiol Behav 101: 494-502, 2010.

41. Csabafi K, Jászberènyi M, Bagosi Z, Liptàk N and Telegdy G: Effects of kisspeptin-13 on the hypothalamic-pituitary-adrenal axis, thermoregulation, anxiety and locomotor activity in rats. Behav Brain Res 241: 56-61, 2013.

42. Kenny R, Dinan T, Cai G and Spencer SJ: Effects of mild calorie restriction on anxiety and hypothalamic-pituitary-adrenal axis responses to stress in the male rat. Physiol Rep 2: e00265, 2014.

43. Sarubin N, Nothdurfter C, Schüle C, Lieb M, Uhr M, Born C, Zimmermannc R, Bühner M, Konopka K, Rupprecht R and Baghai TC: The influence of Hatha yoga as an add-on treatment in major depression on hypothalamic-pituitary-adrenal-axis activity: A randomized trial. J Psychiatr Res 53: 76-83, 2014.

44. McElroy SL, Kotwal R, Malhotra S, Nelson EB, Keck PE and Nemeroff CB: Are mood disorders and obesity related? A review for the mental health professional. J Clin Psychiatry 65: 634-651, 2004.

45. De Kloet ER, Vreugdenhil E, Oitzl MS and Joëls M: Brain corticosteroid receptor balance in health and disease. Endocr Rev 19: 269-301, 1998.

46. de Kloet ER, Sutanto W, van den Berg DT, Carey MP, van Haarst AD, Hornsby CD, Meijer OC, Rots NY and Oitzl MS: Brain mineralocorticoid receptor diversity: Functional implications. J Steroid Biochem Mol Biol 47: 183-190, 1993.

47. de Quervain DJ, Aerni A, Schelling G and Roozendaal B: Glucocorticoids and the regulation of memory in health and disease. Front Neuroendocrinol 30: 358-370, 2009.

48. Zhe D, Fang H and Yuxiu S: Expressions of hippocampal mineralocorticoid receptor (MR) and glucocorticoid receptor (GR) in the single-prolonged stress-rats. Acta Histochem Cytochem 41: 89-95, 2008.

49. de Kloet ER, Oitzl MS and Joëls M: Functional implications of brain corticosteroid receptor diversity. Cell Mol Neurobiol 13: 433-455, 1993. 